\title{
Intrinsic pro-angiogenic status of cystic fibrosis airway epithelial cells
}

\author{
Catherine Verhaeghe ${ }^{\mathrm{a}, 1}$, Sebastien P. Tabruyn ${ }^{\mathrm{b}, 1}$, Cecile Oury ${ }^{\mathrm{a}}$, Vincent Bours ${ }^{\mathrm{a}, *, 2}$, \\ Arjan W. Griffioen b,2 \\ a Unit of Human Genetics, GIGA-Research, University of Liège, Belgium \\ ${ }^{\mathrm{b}}$ Department of Pathology, Angiogenesis Laboratory, GROW, University of Maastricht, The Netherlands
}

Received 22 February 2007

Available online 16 March 2007

\begin{abstract}
Cystic fibrosis is a common genetic disorder characterized by a severe lung inflammation and fibrosis leading to the patient's death. Enhanced angiogenesis in cystic fibrosis (CF) tissue has been suggested, probably caused by the process of inflammation, as similarly described in asthma and chronic bronchitis. The present study demonstrates an intrinsic pro-angiogenic status of cystic fibrosis airway epithelial cells. Microarray experiments showed that CF airway epithelial cells expressed several angiogenic factors such as VEGF-A, VEGF-C, bFGF, and PLGF at higher levels than control cells. These data were confirmed by real-time quantitative PCR and, at the protein level, by ELISA. Conditioned media of these cystic fibrosis cells were able to induce proliferation, migration and sprouting of cultured primary endothelial cells. This report describes for the first time that cystic fibrosis epithelial cells have an intrinsic angiogenic activity. Since excess of angiogenesis is correlated with more severe pulmonary disease, our results could lead to the development of new therapeutic applications.
\end{abstract}

(c) 2007 Elsevier Inc. All rights reserved.

Keywords: Angiogenesis; Cystic fibrosis; Lung Inflammation; VEGF; Endothelial cells; Epithelial cells

Cystic fibrosis (CF) is caused by a mutation in the cystic fibrosis transmembrane conductance regulator (CFTR) gene and is the most common life-shortening genetic disorder among caucasians. While different organs are affected in CF patients, chronic lung disease and fibrosis are the most severe clinical manifestations leading to the patient's

\footnotetext{
is C.V. was supported by a F.R.I.A. ("Fond pour la recherche industrielle et agricole") and by a 'Fondation Leon Fredericq' fellowships. S.P.T. and A.W.G. was supported by a Senter/Novem Fellowship. C.O. is a Research Associate of the F.N.R.S (Belgium).

论 cystic fibrosis airways. Verhaeghe C., Remouchamps C., Vanderplasschen A., Hennuy B., Chariot A., Tabruyn SP, Oury C., Bours V. Biochemical Pharmacology. In Revision.

* Corresponding author. Fax: +324366 8146.

E-mail address: vbours@ulg.ac.be (V. Bours).

${ }^{1}$ These authors contributed equally to this work.

2 These authors share equal responsibility in this work.
}

death [1]. While inflammation is recognized as the driving force leading to fibrosis, increasing evidence also supports the contribution of vascular remodeling in pulmonary fibrosis [2]. Angiogenesis, the formation of new blood vessels from pre-existing ones, is linked to many pathologies characterized by lung inflammation (asthma, chronic obstructive pulmonary disease or idiopathic pulmonary fibrosis) and contributes to maintain a chronic inflammatory state $[3,4]$. New vessels facilitate the diapedesis of inflammatory cells and produce a significant source of pro-inflammatory cytokines. Signs of excessive angiogenesis have been already reported in CF patients who develop highly vascularized nasal polyps requiring surgical resection. In addition, angiogenesis-related complications, such as pulmonary hemorrhage and hemoptysis commonly occur in CF patients [5]. In addition, a previous report has shown higher levels of vascular endothelial growth factor (VEGF) in the serum of CF patients than in other 
pulmonary inflammatory disorders. These higher levels are correlated with a more severe pulmonary disease [6]. The production of VEGF, as well as the induction of angiogenesis, is commonly interpreted as a consequence of the inflammation process.

In this paper, we describe the overproduction of potent pro-angiogenic factors in human epithelial fetal tracheal cells (CF cells), originating from a cystic fibrosis fetus, as compared to control cells. This production is sufficient to stimulate angiogenesis. Our results thus suggest that an intrinsic pro-angiogenic status of CF airway epithelial cells, contributes to enhanced angiogenesis and clinical symptoms.

\section{Materials and methods}

Cell culture. Immortalized human fetal tracheal cells (CFT-2, cell line carrying the homozygous mutation $\Delta \mathrm{F} 508$, and NT-1, control cell line) were previously characterized $[7,8]$. Human umbilical vein endothelial cells (HUVEC) were isolated and cultured as previously described [9]. Only cells from passage 1 to 3 were used for experiments.

Real-time RT-PCR. Real-time PCR was carried out on a TaqMan platform using SYBR Green reagent (Applied Biosystems, Foster city, CA) as described previously [10]. Primers were designed using the Primer Express software (Applied Biosystems). Transcript levels were normalized using the housekeeping gene $\beta_{2}$-microglobulin.

ELISA. Concentrations of VEGF in NT-1 and CFT-2 supernatants were determined by the 'Quantikine human VEGF' immunoassay according to the manufacturer's recommendations (R\&D Systems).

Proliferation assay. HUVECs were seeded at 5000 cells/well in gelatincoated flat-bottomed 96-well tissue culture plates and grown for 3 days in culture medium supplemented with $0.1 \%$ FCS and various volume of NT1 or CFT2 supernatant. On the third day, BrdU was added and incorporation was allowed to occur for $18 \mathrm{~h}$. After harvesting the cells, BrdU incorporation was quantified using the Cell Proliferation Biotrak ELISA system (Amersham Biosciences) according to the manufacturer's recommendations.

Migration assay. Migration assays were carried on as previously described [11]. Briefly, HUVEC cultures were grown to confluency in gelatin-coated 24-well tissue culture plates. A cross-shape wound was made by scratching the monolayer with a plastic tip. Wounded mono- layers were washed with PBS and incubated with fresh culture medium supplemented with $0.1 \%$ FCS and various volume of NT1 or CFT2 supernatant. The wound width was measured microscopically at four different places $0,2,4$, and $6 \mathrm{~h}$ after wounding the culture.

Sprouting assay. HUVEC were suspended in culture medium and seeded on non-adherent plates. The suspended cells integrate into a single spheroid of defined size and cell number. After $24 \mathrm{~h}$ of incubation at $37^{\circ} \mathrm{C}$, the spheroids were embedded in a collagen gel and treated with the different conditioned media. Quantification was performed by digital imaging and calculating length of the spheroid sprouts after $8 \mathrm{~h}$ of incubation with the conditioned media. At least 10 spheroids per group and experiment were analyzed.

Statistical analysis. Data are expressed as mean values \pm SEM and were analyzed by a Student's $t$-test. Differences are considered significant at $p \leqslant 0.05$.

\section{Results}

\section{CF cells differentially express angiogenic genes}

Our previous gene expression profile analysis indicated overexpression of several pro-inflammatory molecules in CF epithelial cells compared to control epithelial cells (see Footnote 记记). The list of differentially regulated genes between $\mathrm{CF}$ and control cells has been further investigated with Ingenuity Systems Pathways (Ingenuity ${ }^{\circledR}$ Systems). In addition to genes described to play a role in both inflammation and angiogenesis, such as interleukin-8 (IL-8), this analysis highlighted up-regulation of numerous pro-angiogenic genes in CF cells, namely basic fibroblast growth factor (bFGF), fibroblast growth factor 5, placental growth factor (PGF), platelet-derived growth factor-C, plateletderived growth factor- $\alpha$ polypeptide, angiopoietin 1, VEGF-A, and VEGF-C. In addition, a well-known antiangiogenic gene, namely thrombospondin-1 (THSP1), was found to be down-regulated in CF compared to control cells.

Differential expression of four selected genes was then confirmed by real-time RT-PCR (Fig. 1A). The expression
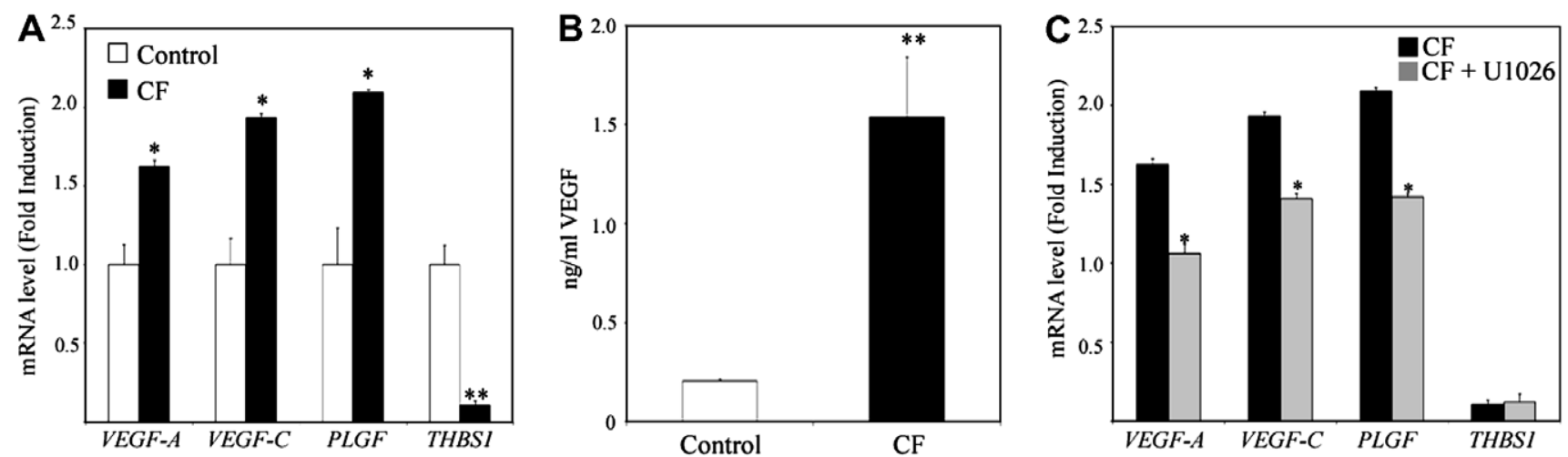

Fig. 1. Validation of differential regulation of angiogenesis-related genes and proteins in CF cells. (A) RNAs from NT-1 and CFT-2 cells were extracted and transcript expression levels of VEGF-A, VEGF-C, PLGF and THSP1 were analyzed by real-time RT-PCR. Values are expressed as fold increase in gene expression in CFT-2 (CF) cells relative to NT-1 (control) cells. Data are normalized by quantification of the $\beta_{2}$ microglobulin transcripts and are representative of at least three distinct experiments. ${ }^{*} p<0.05$ and ${ }^{* *} p<0.005$ vs. NT-1. (B) Quantification by ELISA of VEGF production in supernatants of NT-1 and CFT-2 cells. Data are expressed in ng/ml of conditioned media. ${ }^{* *} p<0.005$ vs. NT-1. (C) CFT-2 cells were treated for 6 h with U1026 $(10 \mu \mathrm{M})$ prior to RNA extraction and determination of transcript expression. Data are expressed as fold increase in gene expression of CF cells relative to control NT-1 cells. ${ }^{*} p<0.05$. Each column represents the means $\pm \operatorname{SD}(n=3)$. 
of genes coding for the pro-angiogenic VEGF-A, VEGF-C, and PLGF was significantly up-regulated in CF cells as compared to control cells (by 1.6-, 1.9-, and 2.0-fold, respectively), while the expression of the anti-angiogenic THSP1 transcript was decreased by about 10 -fold in CF cells in comparison to control cells. ELISA tests confirmed a 8-fold higher production of VEGF-A by CF cells, a protein known for its central role in angiogenesis (Fig. 1B) [12].

\section{Involvement of ERK in pro-angiogenic gene up-regulation in CF cells}

In a previous work, we demonstrated a constitutive AP-1 activation in CF epithelial cells. Furthermore, we demonstrated that ERK1/2 signaling contributes to the overexpression of pro-inflammatory genes (see Footnote 证论). To determine if ERK $1 / 2$ is also involved in the upregulation of the pro-angiogenic genes, $\mathrm{CF}$ cells were treated with the specific inhibitor of MEK1 (ERK kinase), U0126. The expression of the three selected up-regulated genes was significantly reduced in the presence of U1026 (Fig. 1C) while down-regulation of THSP1 was not affected (Fig. 1C). These data suggest that part of the pro-angiogenic status of CF epithelial cells depends on the activation of the ERK1/2 pathway.

\section{CF cells induce proliferation of endothelial cells}

Since CF cells express various potent angiogenic factors, we analyzed the pro-angiogenic properties of conditioned media. Conditioned media from $\mathrm{CF}$ and control epithelial cells were collected and tested for their ability to induce proliferation of HUVECs. Endothelial cells stimulated with various dilutions of $\mathrm{CF}$ cell conditioned media dis-

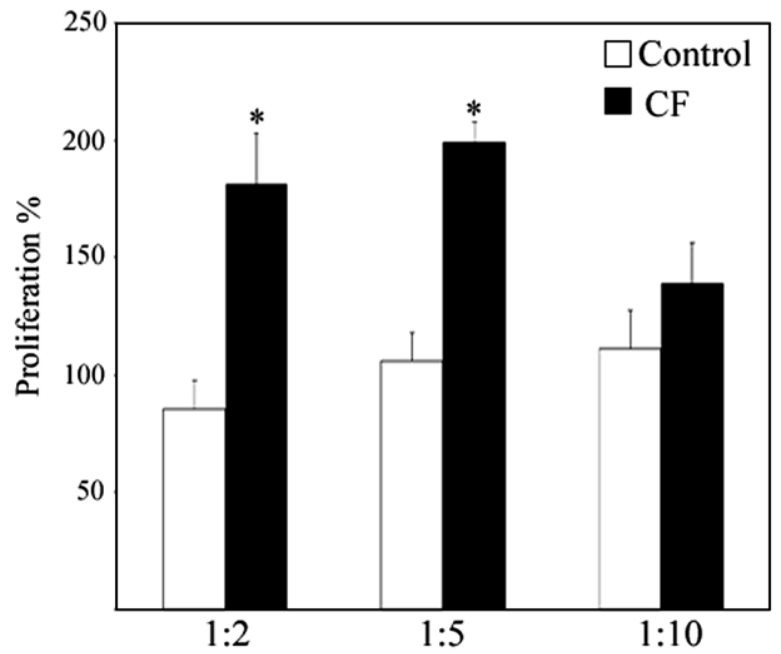

Fig. 2. Induction of endothelial cells proliferation by CF cells. Proliferation of endothelial cells was determined by monitoring DNA synthesis. HUVEC were stimulated for $48 \mathrm{~h}$ with various dilutions of $\mathrm{CF}$ or control conditioned media and assessed for their ability to incorporate BrdU. BrdU incorporation was then measured by ELISA. Each column represents the means $\pm \mathrm{SD}(n=3) .{ }^{*} p<0.05$ vs. NT-1. played a higher proliferation rate than control cells, as measured by incorporation of BrdU (Fig. 2).

\section{CF cells induce migration of endothelial cells}

The effect of conditioned media on endothelial cell migration was then measured by a wound assay. Migration of endothelial cells into the wounded area was significantly increased in the presence of CF conditioned media as compared to control conditioned media. This effect was already visible as early as $2 \mathrm{~h}$ after wounding and was significantly different from the control after 4 and $6 \mathrm{~h}$ (Fig. 3A). In addition, after $6 \mathrm{~h}$, the migration was inhibited by diluting the $\mathrm{CF}$ conditioned media (Fig. 3B), which is indicative of a dose dependent effect.

\section{CF cells induce sprouting of endothelial cells}

HUVEC spheroids seeded into a collagen gel in the presence of angiogenic stimuli have the ability to sprout
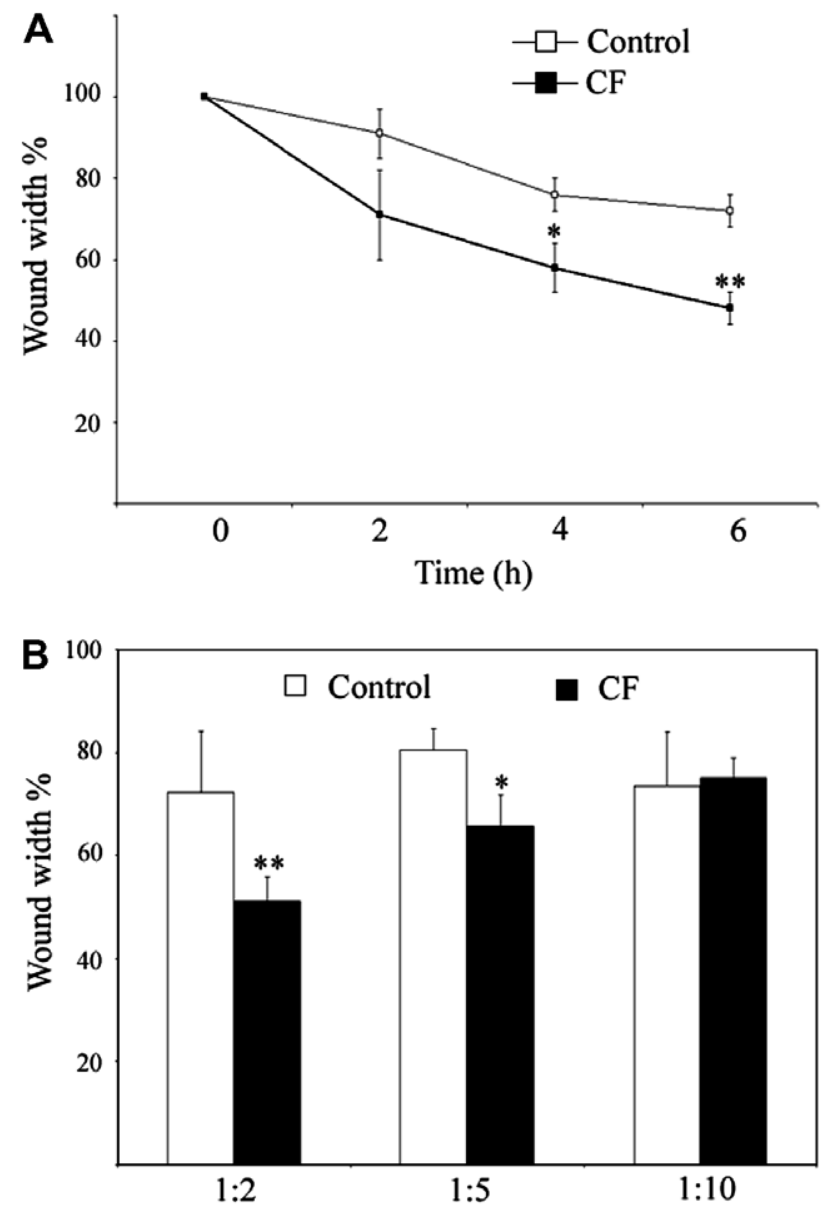

Fig. 3. Induction of endothelial cells migration by CF cells. Migration of endothelial cells was determined by wound assays. Confluent HUVEC cultures were wounded and treated with $\mathrm{CF}$ or control conditioned media. Subsequently, wound width was measured at 2, 4, and $6 \mathrm{~h}$ (A). Wound width of HUVEC cultures treated with different dilutions of conditioned media was also analyzed at $6 \mathrm{~h}$ after wounding (B). Data are representative of at least three distinct experiments. Each bar represents the means $\pm \mathrm{SD}, n=3 .{ }^{*} p<0.05$ and ${ }^{* *} p<0.005$ vs. WT. 

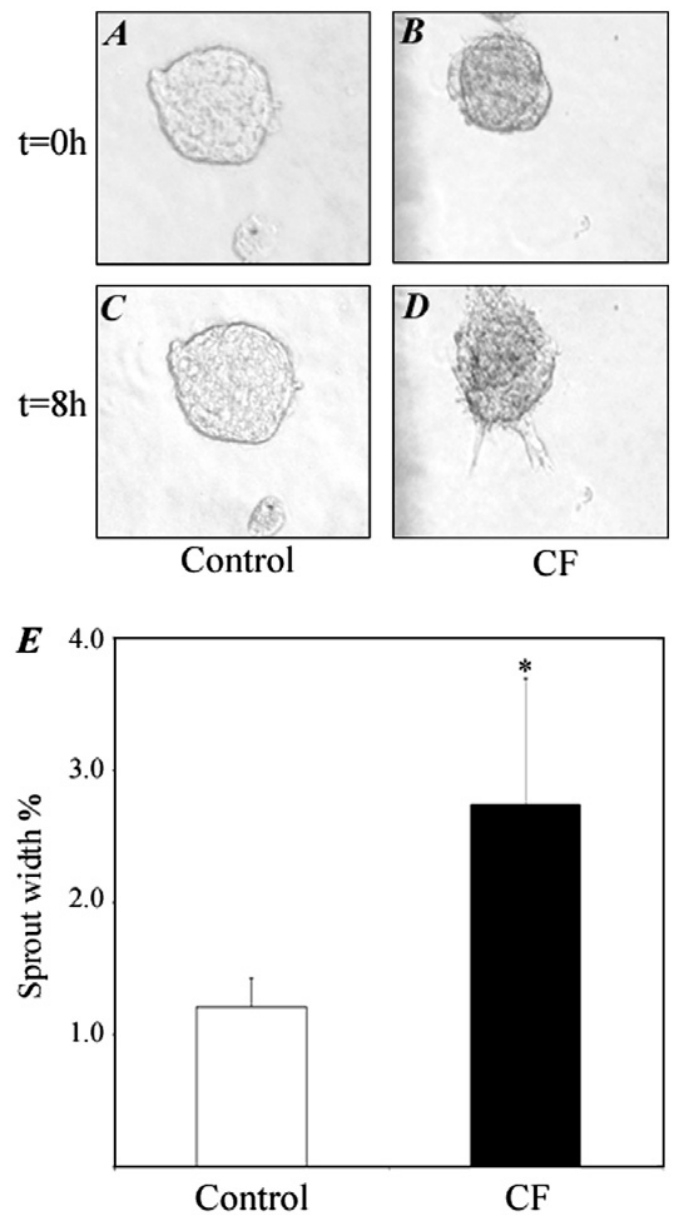

Fig. 4. Induction of endothelial cells sprouting by $\mathrm{CF}$ cells. In vitro angiogenesis was determined using sprouting assay based on collagen embedded HUVEC spheroids. Spheroids were embedded into collagen and photographed before $(\mathrm{A}, \mathrm{B})$ and $8 \mathrm{~h}(\mathrm{C}, \mathrm{D})$ after incubation with WT $(\mathrm{A}-\mathrm{C})$ or $\mathrm{CF}(\mathrm{B}, \mathrm{D})$ cells conditioned media. (E) For each experiment, at least 10 spheroids per group were analyzed and cumulative spheroids length was measured. Data are representative of at least three distinct experiments. Each bar represents the mean $\pm \mathrm{SD}, n=3 .{ }^{*} p<0.05$ vs. WT.

and form three-dimensional capillary-like structures. We investigated the effects of $\mathrm{CF}$ cell conditioned media in a collagen-gel-based sprout assay. Fig. 4A-D show representative pictures of spheroids before (A and $\mathrm{B})$ and after an 8 h-treatment with control (A and C) and CF (B and D) conditioned media. A higher percentage of spheroids was found to sprout in the presence of CF than in the presence of control conditioned media ( $62 \%$ vs. $35 \%$ ). Quantitative assessment of this in vitro angiogenesis assay was achieved by measuring the size of the capillary sprouts out of the spheroids. The cumulative sprout length was found to be significantly increased for the spheroids treated with CF cell conditioned media (Fig. 4E).

\section{Discussion}

Although angiogenesis has been associated with cystic fibrosis, the literature on this subject is really sparse. The only available data described an excess of angiogenesis in CF polyps and elevated levels of VEGF in the serum of cystic fibrosis patients $[5,6]$. Angiogenesis and vascular remodeling are key elements participating in tissue remodeling in various chronic airway inflammatory diseases such as asthma and chronic bronchitis $[4,13]$. Such alterations in the microvasculature largely contribute to the pathophysiology of chronic inflammatory airway diseases. The origin of angiogenesis in the lung of $\mathrm{CF}$ patients is controversial. It was generally considered that the abnormal mucus favors infections and lung colonization by bacteria, which in turn leads to a chronic inflammation and subsequent angiogenesis [14,15]. However, several recent findings from our and other groups suggest that some degree of inflammation could be observed in $\mathrm{CF}$ cells or lungs even before any infection [16-19]. Our results newly show that human epithelial fetal tracheal cells carrying the homozygous CFTR mutation $\Delta \mathrm{F} 508$ display a pro-angiogenic state compared to control epithelial cells. This pro-angiogenic state is characterized by the increased production of VEGF-A but also by the upregulation of at least 10 genes encoding different pro-angiogenic factors such as VEGF-C, PLGF, ANG1, and bFGF. In addition, we observed a downregulation of at least one angiostatic agent, namely thrombospondin-1. These results underline that VEGF is not the only factor involved in the excess of angiogenesis in cystic fibrosis. CF conditioned media were found to induce several angiogenic function of primary endothelial cells such as proliferation, migration and sprouting. These data suggest that $\mathrm{CF}$ epithelial cells could efficiently induce angiogenesis.

Along with the angiogenic process, microvascular remodeling is known to be a key feature of inflammatory diseases [13]. This process involves structural alterations, usually enlargement of arterioles, capillaries or venules. In addition, these remodeled blood vessels have abnormalities in endothelial barrier function and are leaky. The role of the angiopoietin family members has been demonstrated in the vascular remodeling process occurring in asthma and chronic bronchitis [20,21]. Similarly, we found that $\mathrm{CF}$ epithelial cells express more Ang1 and angiopoietin like-2 than control epithelial cells. These results raise the possibility that $\mathrm{CF}$ epithelial cells could initiate or increase the inflammation-related vascular remodeling in $\mathrm{CF}$ patients.

Although there is still a debate about a pre-existing proinflammatory state in CF epithelial cells, our group and others have demonstrated constitutive activation of two important transcription factors that play central role in the onset and persistence of an inflammatory process: NF- $\kappa$ B and AP-1 [16,22-26]. Furthermore, we have shown a role for the ERK1/2 signaling pathway in pro-inflammatory gene expression in CF cells (see Footnote 2 论). In this study, using the NF- $\mathrm{KB}$ inhibitor BAY 11-7085 and the specific inhibitor of MEK1 (ERK kinase), we highlighted that ERK1/2 pathway, but not the NF- $\mathrm{KB}$ pathway (data 
not shown), was involved in the expression of pro-angiogenic genes.

In conclusion, this study reports the ability of $\mathrm{CF}$ epithelial cells to intrinsically promote angiogenesis via the expression of angiogenic factors. Overexpression of different pro-angiogenic molecules by $\mathrm{CF}$ epithelial cells could contribute to the alterations of the microvasculature by increasing angiogenesis but also by inducing microvascular remodeling. Since angiogenesis is correlated with more severe pulmonary diseases, therapies based on angiostatic agents could therefore be efficient to reduce this phenomenon and to decrease the severity of the CF pathology.

\section{Acknowledgments}

The authors are grateful to Dr. J. Capeau (Paris, France) for the gifts of NT-1 and CFT-2 cells.

\section{References}

[1] D.R. Koehler, G.P. Downey, N.B. Sweezey, A.K. Tanswell, J. Hu, Lung inflammation as a therapeutic target in cystic fibrosis, Am. J. Respir. Cell Mol. Biol. 31 (2004) 377-381.

[2] J.W. Wilson, C.F. Robertson, Angiogenesis in paediatric airway disease, Paediatr. Respir. Rev. 3 (2002) 219-229.

[3] P. Carmeliet, Angiogenesis in life, disease and medicine, Nature 438 (2005) 932-936.

[4] M.P. Keane, R.M. Strieter, J.P. Lynch 3rd, J.A. Belperio, Inflammation and angiogenesis in fibrotic lung disease, Semin. Respir. Crit. Care Med. 27 (2006) 589-599.

[5] P.A. Flume, J.R. Yankaskas, M. Ebeling, T. Hulsey, L.L. Clark, Massive hemoptysis in cystic fibrosis, Chest 128 (2005) 729-738.

[6] S.A. McColley, V. Stellmach, S.R. Boas, M. Jain, S.E. Crawford, Serum vascular endothelial growth factor is elevated in cystic fibrosis and decreases with treatment of acute pulmonary exacerbation, Am. J. Respir. Crit. Care Med. 161 (2000) 1877-1880.

[7] M. Lemnaouar, E. Chastre, A. Paul, M. Mergey, D. Veissiere, G. Cherqui, P. Barbry, B. Simon-Bouy, P. Fanen, C. Gespach, et al., Oncogene-mediated propagation of tracheal epithelial cells from two cystic fibrosis fetuses with different mutations. Characterization of CFT-1 and CFT-2 cells in culture, Eur. J. Clin. Invest. 23(1993) 151-160.

[8] S. Medjane, B. Raymond, Y. Wu, L. Touqui, Impact of CFTR DeltaF508 mutation on prostaglandin E2 production and type IIA phospholipase A2 expression by pulmonary epithelial cells, Am. J. Physiol. Lung Cell. Mol. Physiol. 289 (2005) L816-L824.

[9] A.E. Dirkx, M.G. oude Egbrink, K. Castermans, D.W. van der Schaft, V.L. Thijssen, R.P. Dings, L. Kwee, K.H. Mayo, J. Wagstaff, J.C. Bouma-ter Steege, A.W. Griffioen, Anti-angiogenesis therapy can overcome endothelial cell anergy and promote leukocyte-endothelium interactions and infiltration in tumors, FASEB J. 20 (2006) 621-630.

[10] S. Olivier, P. Close, E. Castermans, L. de Leval, S. Tabruyn, A. Chariot, M. Malaise, M.P. Merville, V. Bours, N. Franchimont, Raloxifene-induced myeloma cell apoptosis: a study of nuclear factorkappaB inhibition and gene expression signature, Mol. Pharmacol. 69 (2006) 1615-1623.
[11] R.J. Brandwijk, R.P. Dings, E. van der Linden, K.H. Mayo, V.L. Thijssen, A.W. Griffioen, Anti-angiogenesis and anti-tumor activity of recombinant anginex, Biochem. Biophys. Res. Commun. 349 (2006) 1073-1078.

[12] P. Carmeliet, VEGF as a key mediator of angiogenesis in cancer, Oncology 69 (Suppl. 3) (2005) 4-10.

[13] D.M. McDonald, Angiogenesis and remodeling of airway vasculature in chronic inflammation, Am. J. Respir. Crit. Care Med. 164 (2001) S39-S45.

[14] S.M. Rowe, S. Miller, E.J. Sorscher, Cystic fibrosis, N. Engl. J. Med. 352 (2005) 1992-2001.

[15] C.J. Dakin, A.H. Numa, H. Wang, J.R. Morton, C.C. Vertzyas, R.L. Henry, Inflammation, infection, and pulmonary function in infants and young children with cystic fibrosis, Am. J. Respir. Crit. Care Med. 165 (2002) 904-910.

[16] C. Verhaeghe, K. Delbecque, L. de Leval, C. Oury, V. Bours, Early inflammation in the airways of a cystic fibrosis foetus, J. Cyst. Fibros. (2007).

[17] C. Hubeau, E. Puchelle, D. Gaillard, Distinct pattern of immune cell population in the lung of human fetuses with cystic fibrosis, J. Allergy Clin. Immunol. 108 (2001) 524-529.

[18] M. Rosenfeld, R.L. Gibson, S. McNamara, J. Emerson, J.L. Burns, R. Castile, P. Hiatt, K. McCoy, C.B. Wilson, A. Inglis, A. Smith, T.R. Martin, B.W. Ramsey, Early pulmonary infection, inflammation, and clinical outcomes in infants with cystic fibrosis, Pediatr. Pulmonol. 32 (2001) 356-366.

[19] K. Balough, M. McCubbin, M. Weinberger, W. Smits, R. Ahrens, R. Fick, The relationship between infection and inflammation in the early stages of lung disease from cystic fibrosis, Pediatr. Pulmonol. 20 (1995) 63-70.

[20] F. Baffert, T. Le, G. Thurston, D.M. McDonald, Angiopoietin-1 decreases plasma leakage by reducing number and size of endothelial gaps in venules, Am. J. Physiol. Heart Circ. Physiol. 290 (2006) H107-H118.

[21] K.E. Kim, C.H. Cho, H.Z. Kim, P. Baluk, D.M. McDonald, G.Y. Koh, In vivo actions of angiopoietins on quiescent and remodeling blood and lymphatic vessels in mouse airways and skin, Arterioscler. Thromb. Vasc. Biol. (2006).

[22] A. Knorre, M. Wagner, H.E. Schaefer, W.H. Colledge, H.L. Pahl, DeltaF508-CFTR causes constitutive NF-kappaB activation through an ER-overload response in cystic fibrosis lungs, Biol. Chem. 383 (2002) 271-282.

[23] C. Cigana, E. Nicolis, M. Pasetto, B.M. Assael, P. Melotti, Antiinflammatory effects of azithromycin in cystic fibrosis airway epithelial cells, Biochem. Biophys. Res. Commun. 350 (2006) 977-982.

[24] O. Tabary, S. Escotte, J.P. Couetil, D. Hubert, D. Dusser, E. Puchelle, J. Jacquot, Relationship between IkappaBalpha deficiency, NFkappaB activity and interleukin-8 production in $\mathrm{CF}$ human airway epithelial cells, Pflugers Arch. 443 (Suppl. 1) (2001) S40-S44.

[25] E. DiMango, A.J. Ratner, R. Bryan, S. Tabibi, A. Prince, Activation of NF-kappaB by adherent Pseudomonas aeruginosa in normal and cystic fibrosis respiratory epithelial cells, J. Clin. Invest. 101 (1998) 2598-2605.

[26] A. Venkatakrishnan, A.A. Stecenko, G. King, T.R. Blackwell, K.L. Brigham, J.W. Christman, T.S. Blackwell, Exaggerated activation of nuclear factor-kappaB and altered IkappaB-beta processing in cystic fibrosis bronchial epithelial cells, Am. J. Respir. Cell. Mol. Biol. 23 (2000) 396-403. 